TECHNICAL NOTE

\section{P.A. Brouwer}

T. Bosman

M.A.A. van Walderveen

T. Krings

A.A. Leroux

P.W.A. Willems

\title{
Dynamic 320-Section CT Angiography in Cranial Arteriovenous Shunting Lesions
}

SUMMARY: Novel 320-section CT scanning equipment enables dynamic noninvasive angiographic imaging of the entire cranial vasculature (4D-CTA). We describe this technique and demonstrate its potential in arteriovenous shunting lesions. 4D-CTA imaging resulted in a correct diagnosis, lesion classification, and treatment-strategy selection in 3 patients, compared with CA. We think that 4D-CTA can further reduce the need for CA, sparing the patient the discomfort and risk associated with an invasive procedure.

ABBREVIATIONS: $\mathrm{AVM}=$ arteriovenous malformation; $\mathrm{CTA}=\mathrm{CT}$ angiography; $\mathrm{CA}=$ conventional angiography; DAVF = dural arteriovenous fistula; MIP = maximum intensity projection; $\mathrm{MRA}=\mathrm{MR}$ angiography; $V R=$ volume-rendering

$n$ the diagnosis of cerebral arteriovenous shunting lesions, such as AVMs or DAVFs, it is key to demonstrate the actual shunting (ie, the premature filling of veins). Traditionally, this is achieved by using CA. Unfortunately, CA is relatively expensive and time-consuming and carries a high incidence of silent embolic events ${ }^{1}$ and a small risk of transient or permanent neurologic deterioration. ${ }^{2,3}$

The development of CT scanning equipment with 320 parallel detector arrays has enabled noninvasive dynamic visualization of the entire cranial circulation, maintaining spatial resolution. ${ }^{4}$ We describe $4 \mathrm{D}-\mathrm{CTA}$, adding temporal resolution as the fourth dimension.

The object of this study was to demonstrate the technique of 320-section 4D-CTA and illustrate its potential as a noninvasive alternative to CA in the diagnosis and follow-up of cranial arteriovenous shunting lesions.

\section{Materials and Methods}

\section{D-CTA}

All 4D-CTA examinations were performed by using the Aquilion ONE (Toshiba Medical Systems, Tokyo, Japan), equipped with 320 detector rows, each $0.5 \mathrm{~mm}$ in width, covering $16 \mathrm{~cm}$ of volume per rotation. Using a dual-head power injector, we administered $60 \mathrm{~mL}$ of nonionic contrast medium, followed by $20 \mathrm{~mL}$ of saline. Subsequently, a dynamic acquisition sequence was performed. The first acquisition in this sequence consisted of a single rotation $(80 \mathrm{kVp}, 300$ $\mathrm{mAs}$ ) to generate a noncontrast scan, because the contrast medium had not yet arrived in the area of interest. This would be used as a mask for all subsequent volumes $(80 \mathrm{kVp}, 120 \mathrm{mAs})$, which allowed visualization of the passage of contrast through the vascular bed.

In 2 patients (cases 2 and 3), the timing of the dynamic sequence was optimized by performing a test-bolus acquisition first. This acquisition consisted of the intravenous administration of $20 \mathrm{~mL}$ of

Received March 25, 2009; accepted after revision May 23.

From the Department of Radiology (P.A.B., T.B., M.A.A.v.W.), Leiden University Medical Centre, Leiden, the Netherlands; and Departments of Medical Imaging (T.K., P.W.A.W.) and Neurosurgery (A.A.L.), Toronto Western Hospital, Toronto, Ontario, Canada.

Please address correspondence to Timo Krings, MD, PhD, Department of Neuroradiology, University Health Network, Toronto Western Hospital 3MCL-429, 399 Bathurst St, Toronto, ON, M5T 2S8, Canada; e-mail: Timo.Krings@uhn.on.ca

Indicates open access to non-subscribers at www.ajnr.org

DOI 10.3174/ajnr.A1747 contrast medium followed by $20 \mathrm{~mL}$ of saline, after which a single axial plane, just below the level of the skull base, was imaged once every 2 seconds to detect the arrival of the contrast to the head.

Each protocol generated 22 volumes $(22 \times 320=7040$ images $)$, which were subtracted by using the mask image and stored as 22 DICOM files. The standard scanner software generated the $4 \mathrm{D}$ reconstructions. In principle, the vascular information could be visualized by using a VR or MIP method from any part of the cranium, at any angle, and at any time during contrast passage.

To calculate contrast burden (in $\mathrm{mSv}$ ), we multiplied the doselength product offered by the scanner software by a conversion factor of $0.0021 .^{5}$

\section{CA}

Diagnostic intra-arterial digital subtraction angiography was performed by using standard biplanar fluoroscopy equipment (Toshiba Medical System; or GE Healthcare, Little Chalfont, Buckinghamshire, UK). Following percutaneous access to the femoral artery, a $5 \mathrm{~F}$ diagnostic catheter was used to inject the internal carotid, external carotid, and vertebral arteries on either side. When indicated, selective injections of the occipital, ascending pharyngeal, and internal maxillary arteries were performed.

\section{Case Material}

The study protocol was approved by our institutional research ethics board and informed consent was obtained from the patients involved. Three cases were selected from our initial experience.

\section{Results}

Comparative images selected from the CA and 4D-CTA studies of the 3 selected cases are shown in Figs 1-3.

The cases demonstrate the potential of 4D-CTA to follow a benign DAVF (case 1), recognize cortical venous reflux (case 2 ), and visualize a small AVM with equivocal planar imaging findings (case 3). Although the diagnosis, classification, and treatment strategy in case 2 could have been derived from the 4D-CTA imaging, selective CA clearly elucidated the angioarchitecture of the complex lesion in much more detail.

The 2 patients undergoing test-bolus injections (cases 2 and 3 ) had the largest $4 \mathrm{D}$-CTA radiation burden, which amounted to $5.1 \mathrm{mSv}$ for the entire protocol. In comparison, a double-phased regular cranial CT (eg, 1 phase with and 1 without contrast) produces a radiation burden of $4 \mathrm{mSv}$. 

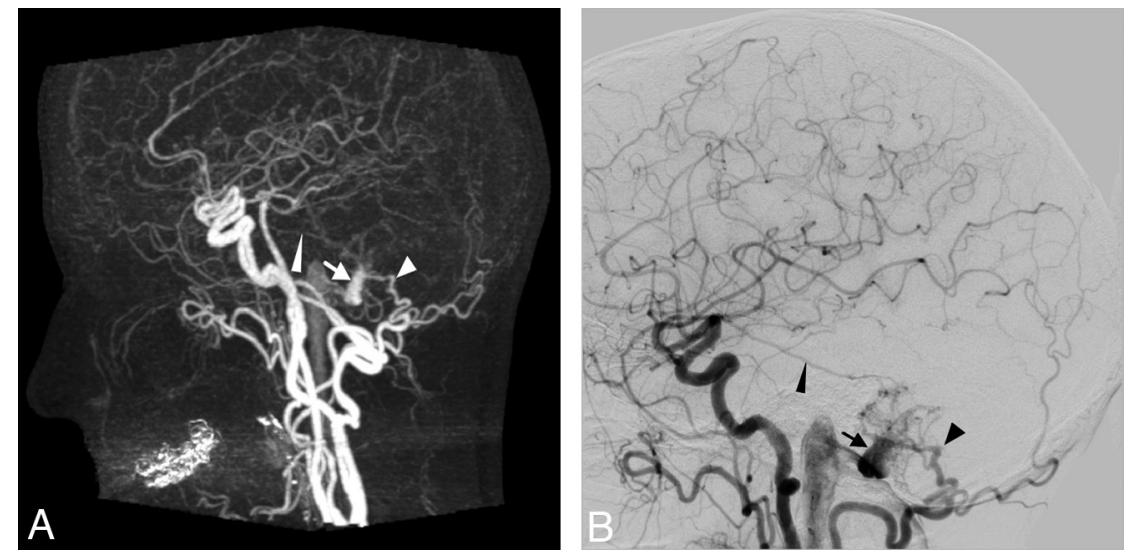

Fig 1. Case 1. Imaging of a 59-year-old woman with an untreated Cognard type I DAVF. A change in her tinnitus led to renewed CA and 4D-CTA imaging. A, Late arterial phase of CTA (MIP, lateral view). B. Late arterial phase of CA (lateral view, common carotid artery injection). Both studies show a branch from the middle meningeal artery (thin arrowhead) and 1 from the occipital artery (arrowhead) feeding a DAVF, which drains into the distal transverse sinus (arrow), without cortical venous reflux.
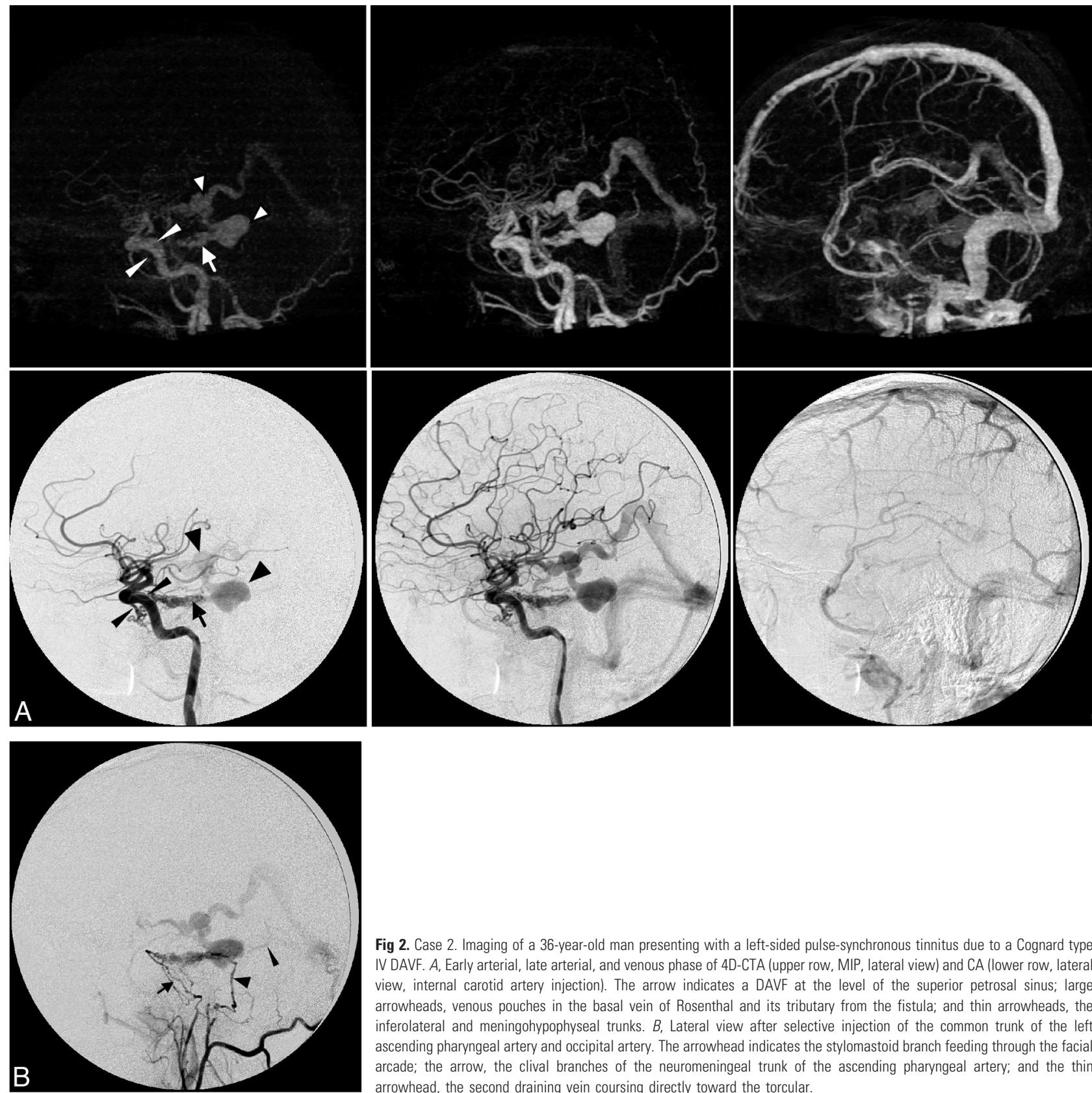

Fig 2. Case 2. Imaging of a 36-year-old man presenting with a left-sided pulse-synchronous tinnitus due to a Cognard type IV DAVF. A, Early arterial, late arterial, and venous phase of 4D-CTA (upper row, MIP, lateral view) and CA (lower row, lateral view, internal carotid artery injection). The arrow indicates a DAVF at the level of the superior petrosal sinus; large arrowheads, venous pouches in the basal vein of Rosenthal and its tributary from the fistula; and thin arrowheads, the inferolateral and meningohypophyseal trunks. $B$, Lateral view after selective injection of the common trunk of the left ascending pharyngeal artery and occipital artery. The arrowhead indicates the stylomastoid branch feeding through the facia arcade; the arrow, the clival branches of the neuromeningeal trunk of the ascending pharyngeal artery; and the thin arrowhead, the second draining vein coursing directly toward the torcular. 


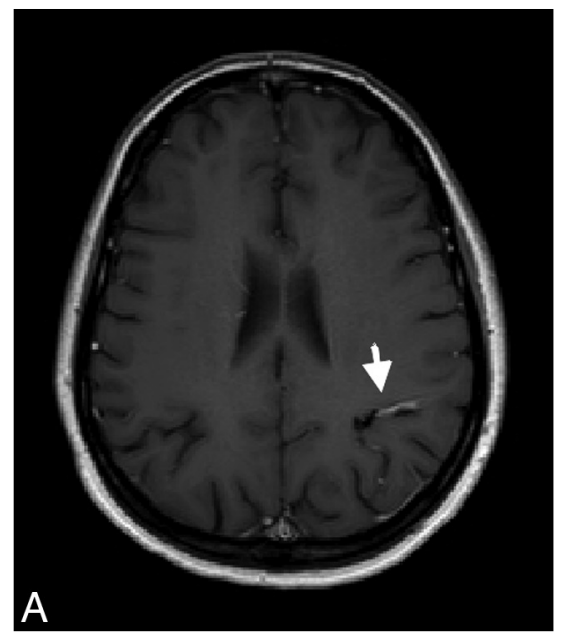

Fig 3. Case 3. Imaging of a 42-year-old woman presenting with new-onset seizures. $A$, Axial contrast-enhanced T1-weighted MR image shows a conspicuous vascular structure (arrow). B, Early arterial, late arterial, and venous phases of CTA (upper row, MIP, lateral view) and CA (lower row, lateral view, internal carotid artery injection). The arrow indicates the feeding artery; the thin arrowhead, the nidus; the large arrowhead, the draining veins; and the long arrow, stenosis in the anterior draining vein.
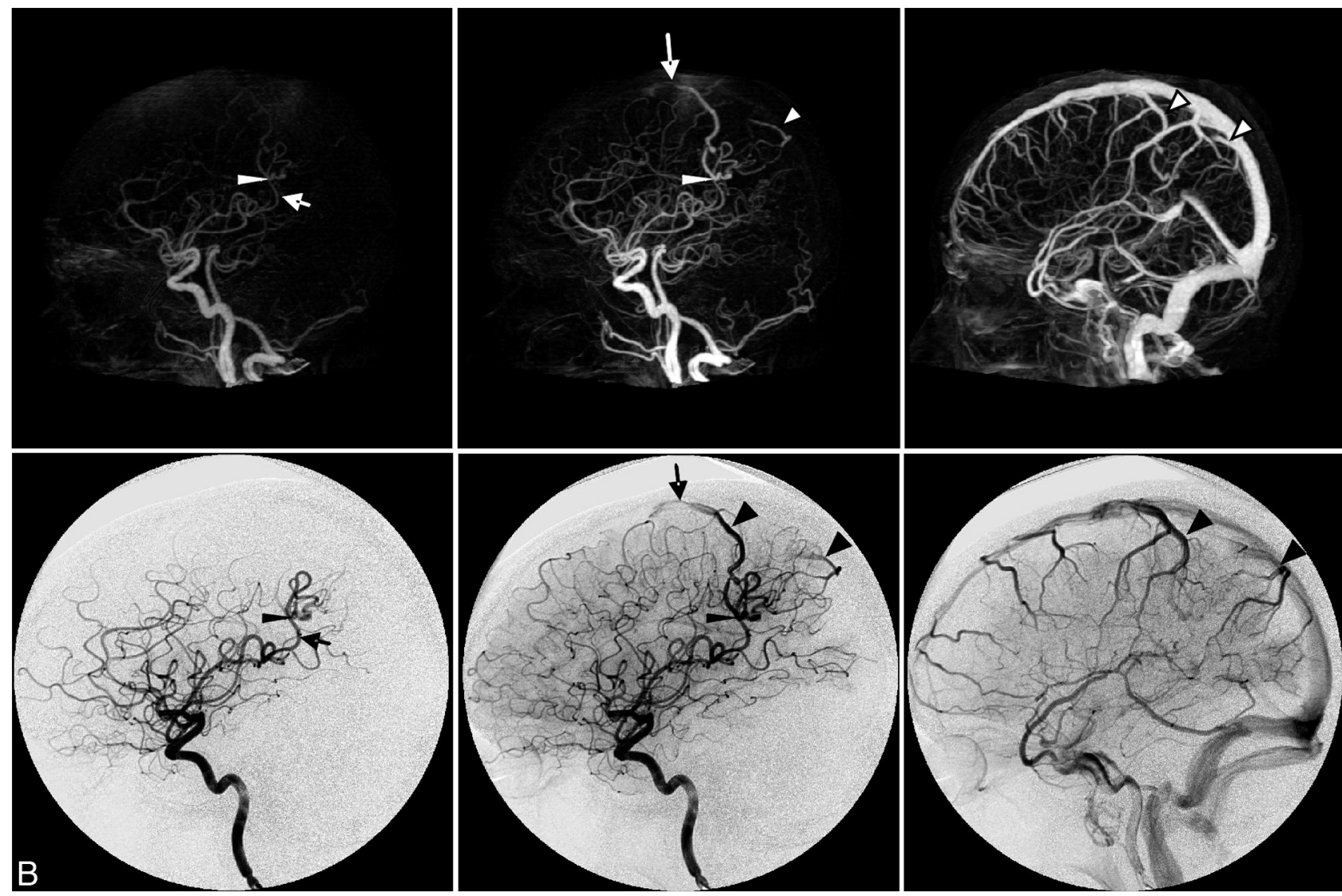

\section{Discussion}

This article describes the first experience with dynamic 320section CT angiography in patients with a cranial arteriovenous shunting lesion. The 3 selected cases illustrate the potential impact of this novel technique in a variety of clinical scenarios.

The images acquired with 4D-CTA differ greatly from CA images. Most important, 4D-CTA features all cranial vascular territories simultaneously, while CA allows selective vessel injection. This may impede the identification of separate tributaries to a shunting lesion, as was demonstrated in case 2. On the other hand, the viewing angles in CA are determined intraprocedurally, while one can visualize $4 \mathrm{D}$-CTA data from any angle during postprocessing. Although VR or MIP reconstructions from $3 \mathrm{D}$ rotational angiography may also be viewed from any angle, these vessel-cast images do not exhibit dynamic information.

With regard to time consumption, in $4 \mathrm{D}$-CTA, the most time will be involved in postprocessing, performed by the CT operator (at present approximately 20-30 minutes). It is, however, considerably less time-consuming than CA for both the patient and radiologist. With regard to radiation and contrast burden, the outcome of the comparison will depend on the extensiveness of the CA study with which the 4D-CTA is compared. However, in case of $4 \mathrm{D}-\mathrm{CTA}$, neither the radiologist nor other supportive staff is exposed to radiation. Last, with regard to patient discomfort and procedure risk, the lack of invasiveness of 4D-CTA certainly compares favorably with CA.

Time-resolved MRA is another noninvasive angiographic 
imaging technique, which may be used as an alternative to CA. ${ }^{6}$ Although we have not yet compared 4D-CTA with timeresolved MRA, the temporal and spatial resolution of our $4 \mathrm{D}$ CTA protocol ( 1 second per volume, voxel size of $0.5 \times 0.5 \times$ $0.5 \mathrm{~mm}$ ) compares favorably with those reported for timeresolved MRA ( 1.7 seconds per volume, voxel size of $1 \times 1 \times$ $2 \mathrm{~mm})^{6}$

For the diagnosis of shunting lesions, we thought that our current protocol offered a good compromise between temporal resolution, signal-to-noise ratio, and radiation burden. The application of 320-section 4D-CTA, however, is not necessarily restricted to the demonstration of early venous filling. Using 0.1-second reconstruction intervals in the postprocessing of a 4D-CTA dataset, we recently demonstrated inhomogeneous aneurysm pulsatility related to the subsequent growth of the aneurysm, ${ }^{7}$ and the generation of CT perfusion images using a similar protocol. ${ }^{8} \mathrm{New}$ dynamic 4D-CTA protocols will likely be developed, including higher gantry rotation speeds ( $\leq 3 \mathrm{~Hz}$ with current equipment), as different research and clinical problems are addressed.

\section{Conclusions}

We believe that 4D-CTA, a noninvasive imaging technique for the entire cranial vasculature with high spatial and temporal resolution, is especially suitable for the diagnosis and classification of arteriovenous shunt surgery lesions, the selection of an appropriate treatment strategy for such lesions, and their follow-up imaging. Future prospective series will be necessary to support these preliminary conclusions.

\section{Acknowledgments}

We thank Gail Reintamm, nurse coordinator, for her organizational assistance; Eric Salomon and Joost Roelofs, CT technologists, for their assistance with protocoling, performing, and postprocessing $4 \mathrm{D}$ CTAs; and Joerg Blobel, Chief of Clinical Science (CT Systems Division) at Toshiba Medical Systems Corporation, for his assistance with the $4 \mathrm{D}$-CTA equipment.

\section{References}

1. Bendszus M, Koltzenburg M, Burger R, et al. Silent embolism in diagnostic cerebral angiography and neurointerventional procedures: a prospective study. Lancet 1999;354:1594-97

2. Willinsky RA, Taylor SM, TerBrugge K, et al. Neurologic complications of cerebral angiography: prospective analysis of 2,899 procedures and review of the literature. Radiology 2003;227:522-28

3. Kaufmann TJ, Huston J 3rd, Mandrekar JN, et al. Complications of diagnostic cerebral angiography: evaluation of 19,826 consecutive patients. Radiology 2007;243:812-19

4. Klingebiel R, Siebert E, Diekmann S, et al. 4-D imaging in cerebrovascular disorders by using 320-slice CT: feasibility and preliminary clinical experience. Acad Radiol 2009;16:123-29

5. Thomas KE, Wang B. Age-specific effective doses for pediatric MSCT examinations at a large children's hospital using DLP conversion coefficients: a simple estimation method. Pediatr Radiol 2008;38:645-56

6. Taschner CA, Gieseke J, Le Thuc V, et al. Intracranial arteriovenous malformation: time-resolved contrast-enhanced MR angiography with combination of parallel imaging, keyhole acquisition, and k-space sampling techniques at 1.5 T. Radiology 2008;246:871-79

7. Krings T, Willems PWA, Barfett J, et al. Pulsatility of an intracavernous aneurysm demonstrated by dynamic 320-detector row CTA at high temporal resolution. Cen Eur Neurosurg 2009;70:214-18

8. Salomon EJ, Barfett J, Willems PWA, et al. Dynamic CT angiography and CT perfusion employing a 320-detector row CT: protocol and current clinical applications. Clinical Neuroradiology 2009;19:187-96 